\title{
FORESIGHT: A GRAPHICALLY BASED APPROACH TO MODELING CONSTRUCTION PROCESSES
}

\author{
Ian Flood \\ Rinker School \\ College of Design, Construction and Planning \\ University of Florida \\ Gainesville, Fl 32611-5703, USA
}

\begin{abstract}
Modeling is an essential part of construction project planning and control. Most modeling exercises use the Critical Path Method (CPM) since it is simple to use and versatile, despite its lack of versatility. Almost all other modeling techniques are aimed at specialized types of construction work, such as linear scheduling which is used for modeling work that progresses along a line. Discrete-event simulation, while extremely versatile, lacks the simplicity in use of CPM and so has not been widely adopted within the industry. This paper goes back to first principles, identifying the needs of construction project planning and how existing tools meet (or fail to meet) these requirements. Based on this, it proposes a new modeling paradigm, Foresight, better suited to contemporary construction project planning. The principles of the method and its relative merits are demonstrated relative to conventional simulation in a series of construction case studies.
\end{abstract}

\section{INTRODUCTION}

The last 100 years have seen the development and adaptation of a wide range of methods for modeling construction processes. An analysis of the genealogy (Flood et al. 2006) of these tools shows that they can be grouped into three main categories: the Critical Path Methods (CPM); the linear scheduling techniques; and process simulation. Most other tools are either an enhancement or an integration of these methods. Even 4D-CAD and nD-CAD methods (Koo \& Fischer 2000; Issa et al. 2003), where one of the dimensions is time, are strictly CPM models hybridized with 3D-CAD for visualization purposes.

Each of the three main groups of modeling method are, unfortunately, only relevant to a restricted range of construction planning problems. The CPM methods (the most popular class of planning tool) are well suited to modeling projects at a relatively general level of detail, but are limited in terms of the types of interactions they can consider between tasks (Harris \& Ioannou 1998). Moreover, CPM models can become unduly complicated when used to model repetitive processes, and provide little understanding of the interactions between repetitive tasks. When presented in Gantt Chart format, a CPM model provides some visual insight into how a system's logic affects its performance (thus suggesting more optimal ways of executing work) but this is limited to event-based logical dependencies and their impact on time-wise performance.

Linear scheduling, on the other hand, is targeted at projects where there is repetition at a high level, such as high-rise, tunneling, and highway construction work (see, for example, Matilla and Abraham (1998)). These models are very easy to understand and represent the system's logic and its performance within a single framework. Consequently, they provide great visual insight into better ways of conducting a project to improve performance, and factors to monitor when work is underway to help ensure it

proceeds efficiently. For example, they show in graphic form how the relative progress of repetitive tasks can lead to conflict, both in terms of time and physical work completed. However, linear schedules can- 
not be used at all to model non-repetitive work, and they include some simplistic assumptions which often make it difficult to model real-world repetitive processes. For example, velocity diagrams (a linear scheduling technique) cannot easily represent operations that follow different paths, such as two underground utility lines that interact at a cross-over point but otherwise follow different routes.

Finally, simulation (see, for example, Halpin and Woodhead (1976); Sawhney et al. (1998); Hajjar and AbouRizk (2002)) is very versatile in that it can in principle model any type of interaction between tasks and any type of construction process (including repetitive and non-repetitive work). However, the effort involved in defining and validating a simulation model means that in practical terms it is best suited to systems that cannot be modeled sufficiently accurately using CPM or linear scheduling. In addition, simulation models provide no visual indication of how a system's logic determines its performance. That is, performance is an output from the model after it has been fully developed; it is not an integral part of the model and therefore its dependence on the model's logic is not directly apparent.

Most projects include a variety of processes some of which may be modeled satisfactorily using CPM while others may be more appropriately represented by linear scheduling or simulation. However, it is not practical to expect planners to employ more than one modeling method to plan and monitor a project. In any case, using several tools that are not fully compatible makes it impossible to seek a globally optimal solution to a planning problem. On the other hand, the alternative approach of using one tool to represent all situations (typically CPM) compromises our ability to plan and control work optimally.

Ideally, what is needed is a single tool that is well suited to modeling the broad spectrum of repetitive and non-repetitive construction work, that is highly versatile, provides insight into better ways of organizing work, and is easy to use. This paper goes back to basics and proposes a new modeling paradigm, Foresight, that addresses the above issues. Section 2 introduces the principles of the Foresight modeling system. Sections 3 to 6 provide five case studies demonstrating the application of Foresight to construction projects that would otherwise best be modeled using discrete-event simulation, linear scheduling, and CPM, respectively.

\section{FORESIGHT MODELING ELEMENTS}

The goal in developing the new approach to modeling was to attain the simplicity of CPM, visual insight of linear scheduling, and the modeling versatility of simulation. In addition, hierarchical structuring of a model (see, for example, Huber et al. (1990) and Ceric (1994)) and interactive development of a model were identified as requisite attributes of the new approach since they facilitate model development and aid understanding of the organization and behavior of a system. The three principle concepts of the Foresight modeling approach are as follows and illustrated in Figure 1:

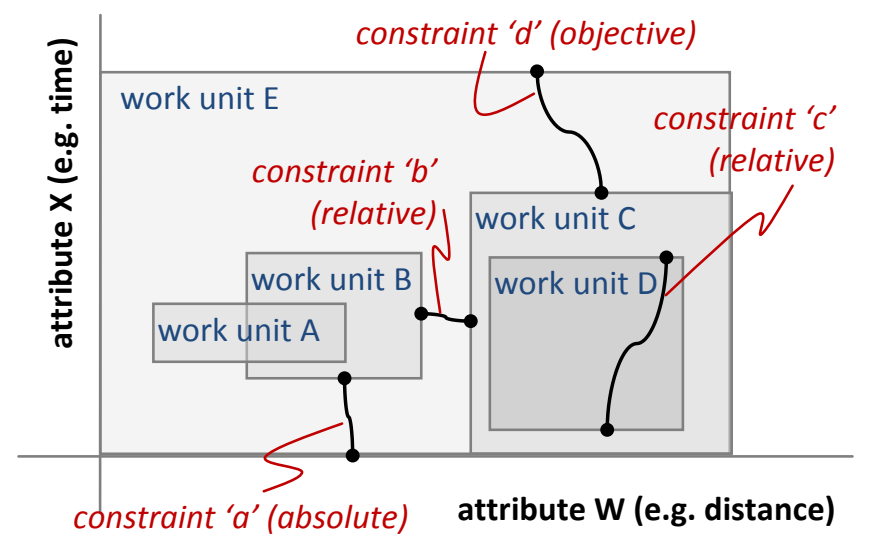

Figure 1: Schematic illustrating the three principle concepts of Foresight 


\section{Flood}

1. Attribute Space. This is the environment within which the model of the process exists. Each dimension defining this space represents a different attribute involved in the execution of the process, such as time, cost, excavators, skilled labor, number of repetitions of an item of work, permits to perform work, and materials. The attributes that make-up this space are resources that need to have their usage monitored, resources that could have a significant impact on performance of the process, and variables that will be used to measure performance.

2. Work Units. These are elements that represent specific items of work that need to be completed as part of the project. They are represented by a bounded region within the attribute space. A unit can represent work at a high level (such as 'Construct Structural System'), a low level (such as 'Erect Column $X^{\prime}$ ) or any intermediate level. Collectively, the work units must represent all work of interest but should not represent any item of work more than once. Most if not all work units will exist in a subset of the dimensions of the attribute space, although normally they will all exist at least within the time dimension. A work unit could in principle take on any form, but for the purposes of this paper they will be limited to rectangular shapes (or hyper-rectangular shapes in the case that they exist within more than 2 dimensions of the attribute space).

3. Constraints and Objectives. Constraints define the relationships between the work units and the attribute space, either directly with the attribute space (such as constraint ' $a$ ' in Figure 1) or indirectly via relationships with other work units (such as constraints ' $b$ ', 'c', and 'd' in Figure 1). These constraints effectively define the location of the edges of the work units. A constraint can be any functional relationship between the borders of the work units and/or the space within which they exist. Practical examples include: (i) ensuring that crews at different work units maintain a safe working distance; (ii) ensuring that the demand for resources never exceeds the number available; (iii) determining the duration for a task based on the number of times it has already been repeated, and (iv) ensuring that idle time for a task is kept to a minimum. The objectives are the specific goals of the planning study, such as to maximize profits or to complete work by a deadline (such as constraint ' $d$ ' in Figure 1). Fundamentally, they are the same thing as constraints, albeit at a higher level of significance, and therefore are treated as such within the proposed new modeling system.

Note that work units can by implication be nested within other work units (such as work unit ' $D$ ' in Figure 1 which is shown to be within work unit ' $\mathrm{C}$ '), or overlap with each other (such as work units ' $A$ ' and ' $\mathrm{B}$ '). Nesting of work units can be defined explicitly, allowing the model to be understood at different levels of abstraction, increasing its readability, reducing the likelihood of errors in the design of the model, and reducing the amount of work required to define and update a model.

A specification of Foresight is that model development be implemented interactively. That is, the visual presentation of a model is updated and all constraints are resolved as the work units and constraints are either edited or added to the model. This way, the modeler can see immediately the impact of any changes or additions that are made. Another point to note is that these models are presented as a plot of the work units within at least two dimensions of the attribute space. This form of presentation allows the progress of work to be visualized within the model's functional structure. This is an extrapolation of the way in which linear scheduling models are presented, and has the advantage of allowing the user to visualize directly how the performance of the model is dependent on its structure. These points will be illustrated in the following three example applications.

It should be noted that Foresight is, strictly speaking, a simulation system in that it requires the use of a three-phase simulation algorithm to resolve its constraints.

\section{EXAMPLE EARTHMOVING SYSTEMS}

The earthmoving system has become one of the classic problems used to introduce and demonstrate the principles of construction simulation. In this section, a range of such systems (in terms of logical complexity) will be considered and compared with CYCLONE, the most widely used and familiar method of construction simulation modeling (Halpin \& Woodhead, 1976). Figure 2 shows the CYCLONE represen- 
tation of a simple excavation system comprising 2 scrapers of 25 cu-m capacity each and 1 bulldozer used to push-load the scrapers. Part (a) of this figure shows the CYCLONE diagram which is a logical representation of the processes involved in the operation, while part (b) shows typical time-wise output from the model measured at the dump activity.

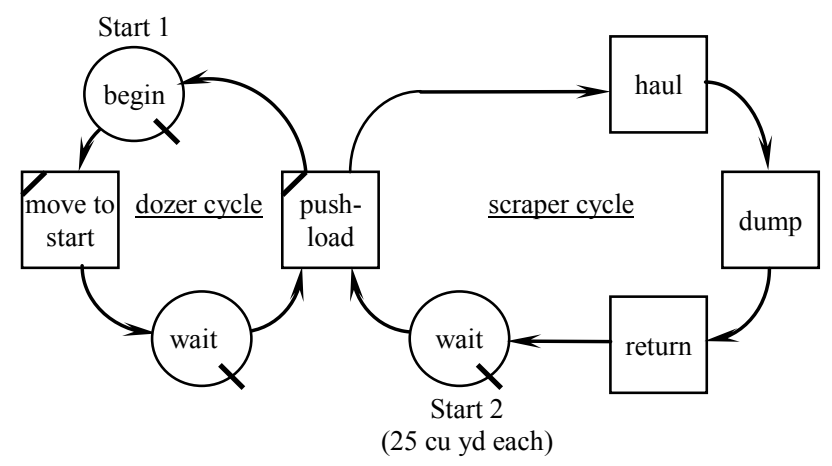

(a) CYCLONE diagram

\section{cu-m dirt}

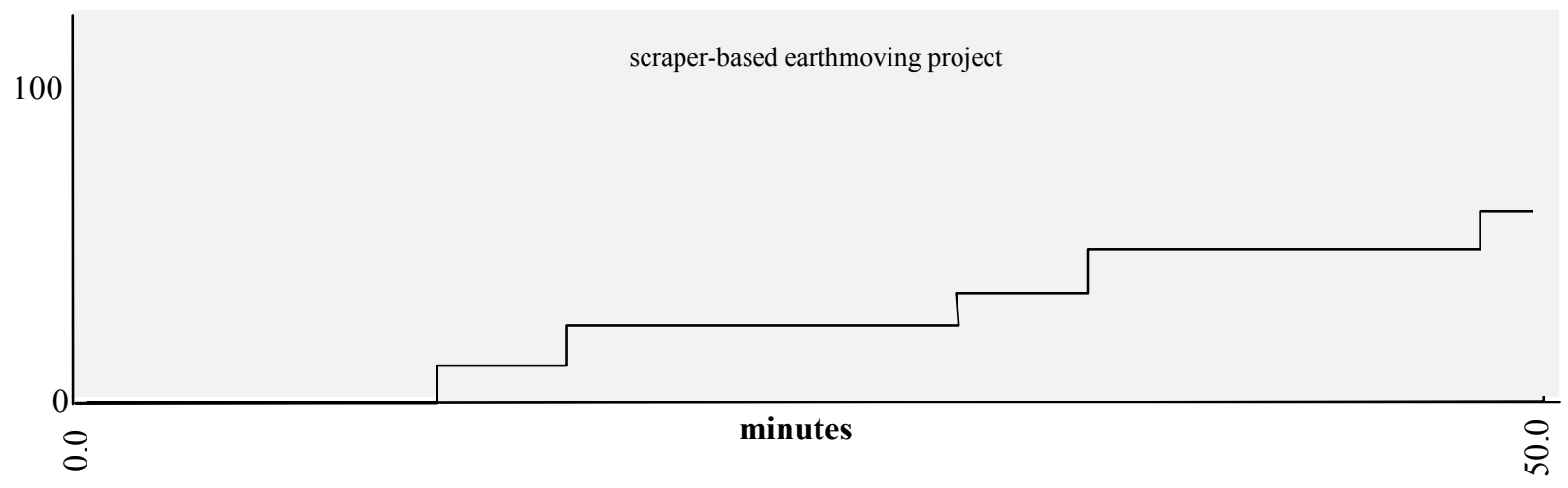

(b) typical progress measured at the dump activity (first 50 minutes)

Figure 2: CYCLONE based simulation modeling of a scraper-based earthmoving system

Figure 3 shows the Foresight equivalent model of the above earthmoving system. Part (a) of Figure 3 shows the hierarchical structure of the model while part (b) shows the model in its normal format with both the model structure and the performance of the system integrated over time. Several important differences between CYCLONE and Foresight can be understood by comparing the model representations of Figures 2 and 3. First, it should be understood that CYCLONE requires the complete logic of the model (as represented by the CYCLONE diagram of Figure 2(a)) to be finalized before the system's performance can be predicted in a simulation run (such as represented by Figure 2(b)). In contrast, the Foresight model integrates the structure and logic of the model and the estimated performance of the system within a single format (as represented by Figure 3(b)). This gives Foresight a couple of significant advantages. First, as elements are added to the model and its parameters altered, the impact of these edits on the estimated performance of the system are seen immediately - the model does not have to be completed before the simulation results are produced. This is a similar advantage to that seen in other graphically based planning tools such as Linear Schedules. The second advantage is that in a Foresight model, the way in which the logic and structure of the model affect the performance of the system is directly visible, which in turn assists in the optimization of the design of the system (this point will be illustrated later in a case study of a sewer-tunneling operation). 


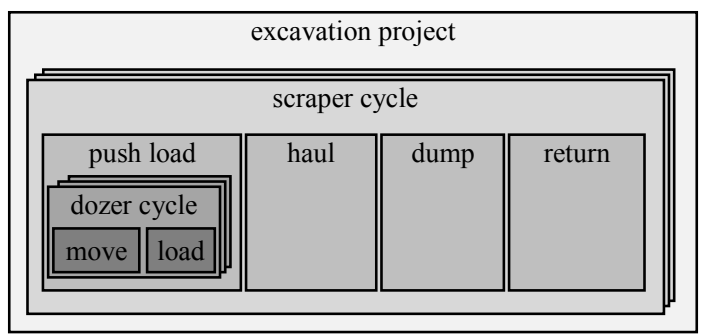

(a) hierarchical structure

\section{cu-m dirt}

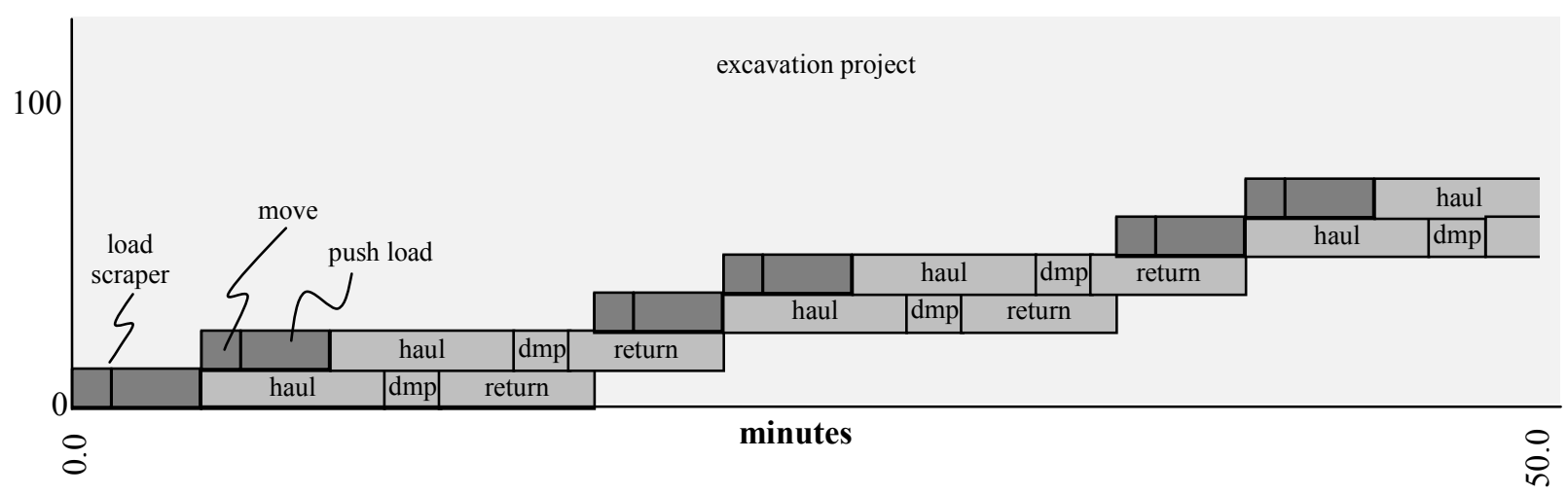

(b) integrated model structure and production

Figure 3: Foresight based simulation modeling of a scraper-based earthmoving system

A slightly more complicated earthmoving system (in modeling logic terms) comprises an excavator used to load dump trucks. The additional complexity arises since the excavator must execute several cycles in order to load a truck; compare this to the scraper-based earthmoving system where the bulldozer was only required to execute one cycle for each scraper cycle. Figure 4 shows the CYCLONE representation of this system for a situation where there are three trucks (each of 5 cu-yds capacity), and one excavator (with a 1 cu-yd bucket). The excavator must therefore perform five cycles to load a truck.

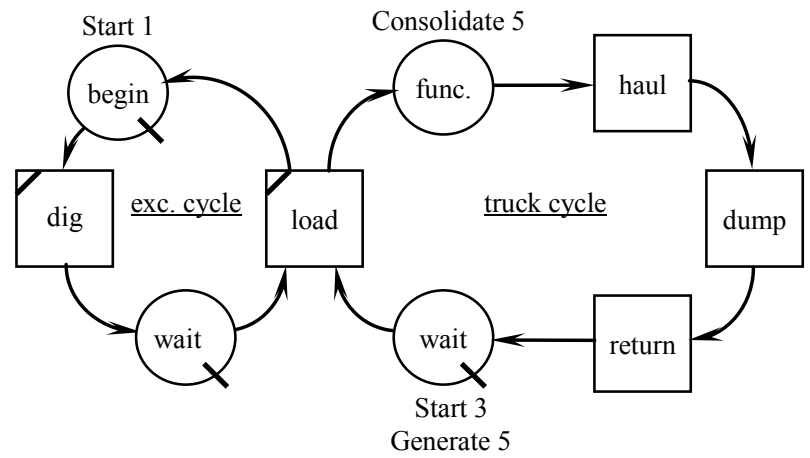

Figure 4: CYCLONE diagram of a dump truck based earthmoving system

The Foresight representation of this system is presented in Figure 5. Part (a) of this figure shows the hierarchical form of the model, whereas part (b) shows the model in its normal format with production integrated into the structure. Figures 5 (c) and (d) show the model using the variables trucks versus time, and excavators versus time respectively. Figure 5(c) only shows the model to the $2^{\text {nd }}$ level in its hierarchy, even though the truck activities go down to the $3^{\text {rd }}$ level, to allow a more generalized understand- 


\section{Flood}

ing of its performance. Likewise, Figure 5(d) shows the activities of the excavator down to the $4^{\text {th }}$ rather than $5^{\text {th }}$ level. These plots effectively show the demand for these productive resources over time, indicating any idle time and thus possible imbalance in the resource combinations.

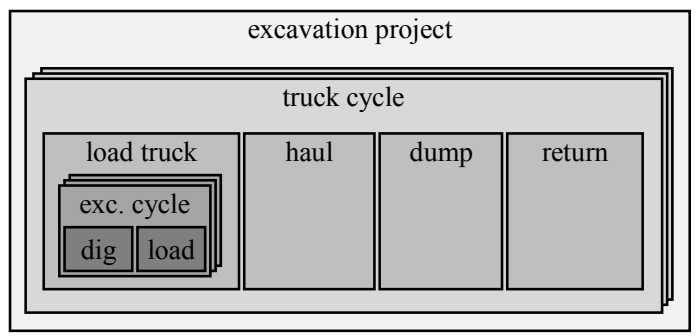

(a) hierarchical structure

\section{cu-m dirt}

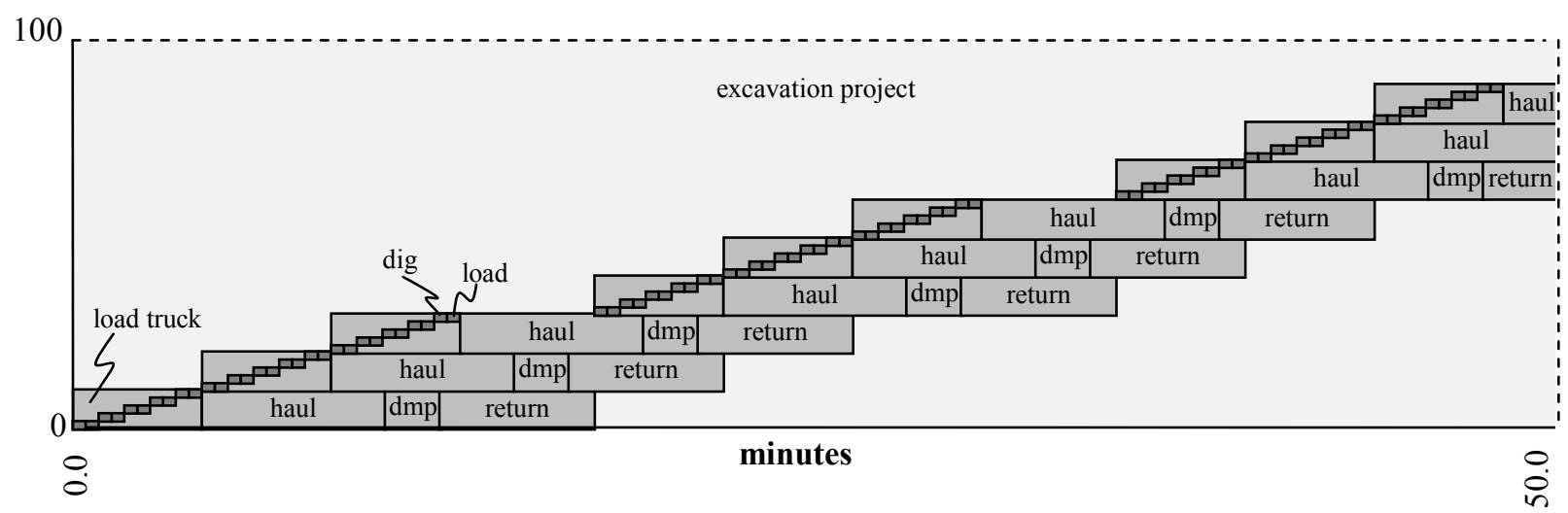

(b) integrated model structure and production

trucks

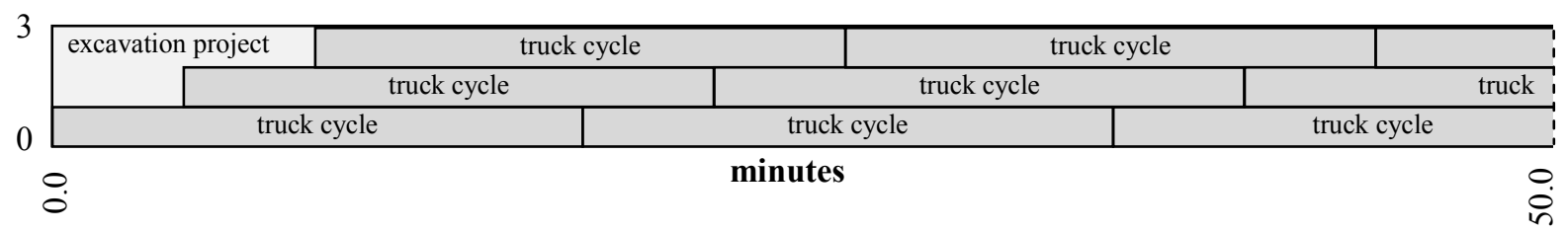

(c) truck demand plotted against time ( $2^{\text {nd }}$ level in model hierarchy)

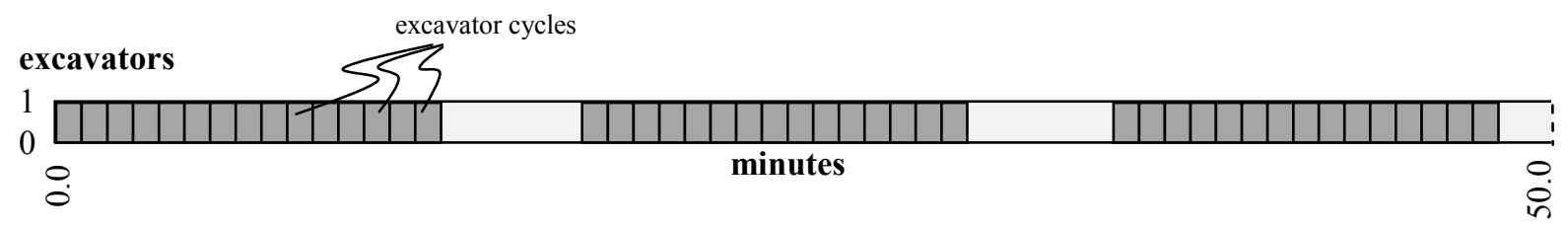

(d) excavator demand plotted against time ( $4^{\text {th }}$ level in model hierarchy)

Figure 5: Foresight model of a dump truck based earthmoving system

\section{SEWER TUNNELING SYSTEM}

The second study is concerned with modeling the construction of a $2 \mathrm{~m}$ internal diameter sewer, where tunneling is through clay and the lining is formed from concrete ring segments. The example is used to illustrate the steps in developing a Foresight model for a relatively complex process. 


\section{Flood}

A component oriented approach should be adopted when developing a Foresight model, such that each work unit represents the construction of a physical component or sub-component of the facility under construction. A top-down, hierarchical approach is an effective strategy for developing these models, starting with the highest level component (the complete facility) and then breaking it down into its constituent components. For the tunnel project, the first two levels of breakdown are shown in Figure 6 . The second level work units here are: excavation representing the cutting of the tunnel; concrete lining which comprises concrete ring segments grouted in place (each $1 \mathrm{~m}$ in length); and light track used to carry a manually propelled train used for removal of spoil and delivery of concrete ring segments (laid in $3 \mathrm{~m}$ lengths).

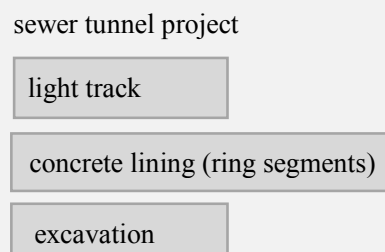

Figure 6: Two levels of work units for the Foresight sewer-tunnel model

At the highest level, the process will repeat itself every $3 \mathrm{~m}$ (the length of the track), and so the model can be more conveniently represented with a work unit representing the construction of a $3 \mathrm{~m}$ length of tunnel that is repeated until the tunnel is completed. Likewise, construction of $1 \mathrm{~m}$ lined sections of the tunnel must be repeated 3 times to complete a $3 \mathrm{~m}$ length of tunnel. This is shown schematically in Figure 7 (a) without imposition of any constraints. From this figure it can be seen that the work units excavation and concrete lining are performed for a $1 \mathrm{~m}$ length of tunnel. The work unit representing this $1 \mathrm{~m}$ lined section is repeated until a $3 \mathrm{~m}$ length of tunnel has been excavated and lined, after which the light track for that $3 \mathrm{~m}$ section is laid. This completes a $3 \mathrm{~m}$ section of the tunnel. The work unit representing the $3 \mathrm{~m}$ section is repeated until the tunnel is complete. The work units, light track, concrete lining, and, excavation are further broken down to a level of detail considered appropriate for the analysis to be performed using the model. In this case, Figure 7(a) shows up to seven levels of breakdown, with the deepest being the dig and load work units nested within the excavation work unit.

Addition of constraints can occur as work units are added to the model. The result of this is shown in Figure 7(b), plotting time against tunnel length for the first 3 meters of the project. In this case, the main constraints are as follows:

- The work units representing $3 \mathrm{~m}$ tunnel sections are positioned serially both in the time and tunnel length dimensions.

- The work unit representing the sewer tunnel project extends in the tunnel length direction to a value equal to the tunnel length.

- The 3 m tunnel section work units start at the left side of the sewer tunnel project work unit and extend all the way to (but not beyond) the right side of the sewer tunnel project work unit.

- The $1 \mathrm{~m}$ lined section work units are positioned serially both in the time and tunnel length dimensions.

- The $1 \mathrm{~m}$ lined section work units span from the left to right side of their $3 \mathrm{~m}$ tunnel section work unit.

- The work units excavation and concrete lining are positioned sequentially in the time dimension. 


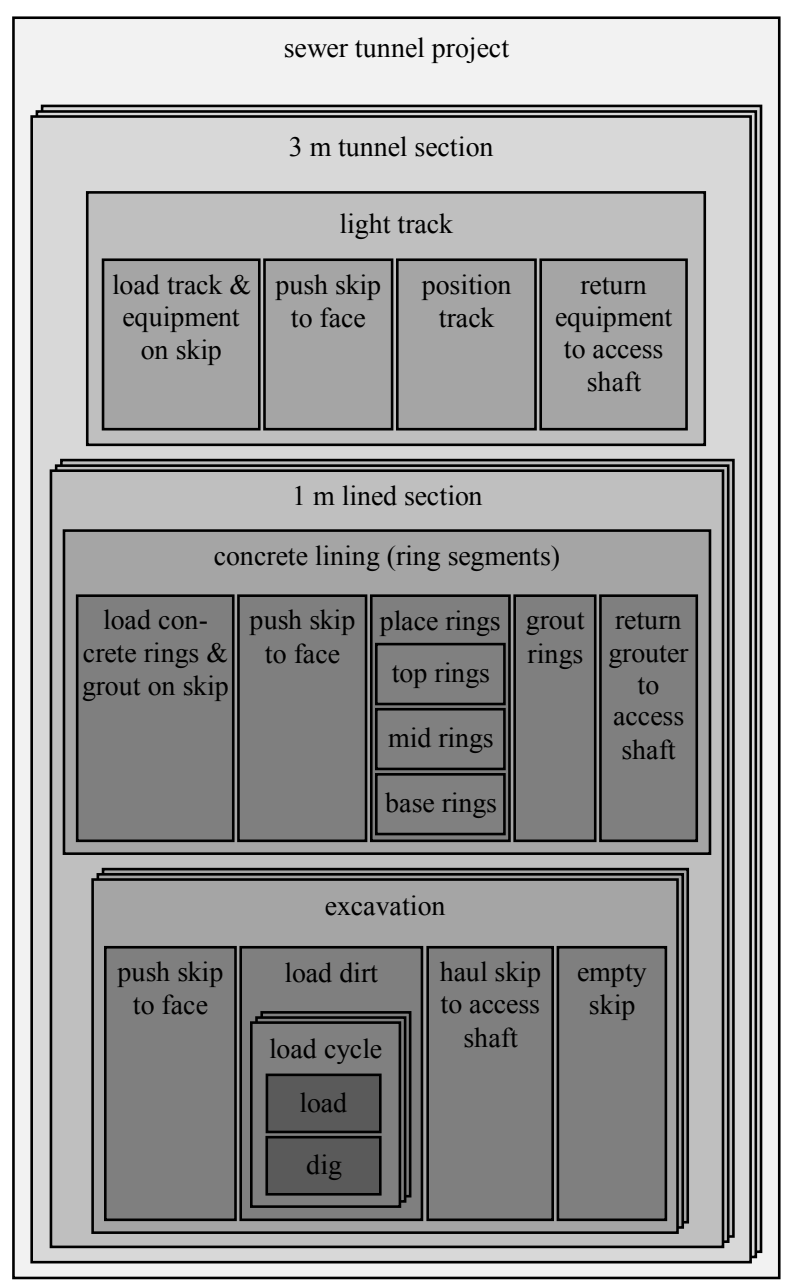

(a) model hierarchy

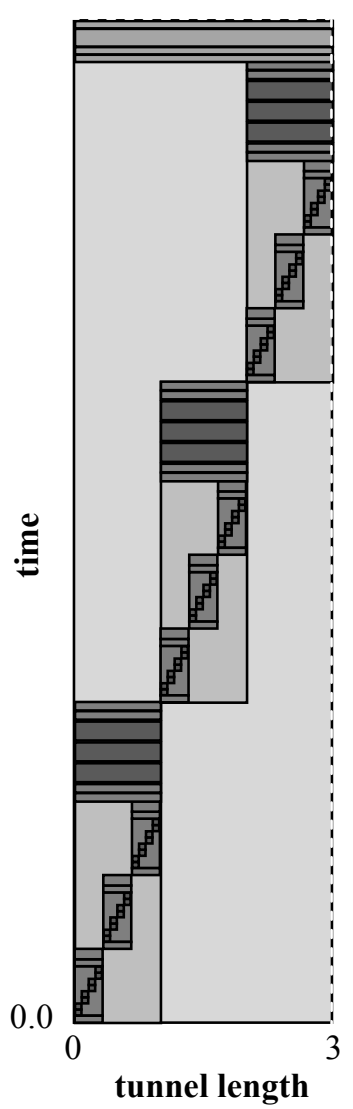

(b) progress over first 3 meters

Figure 7: Foresight Model of a Sewer-Tunnel Operation

Figure 8 shows the model for the first 30 meters of tunnel. For readability, the model is only shown to a level of breakdown that shows the excavation, concrete lining and light track work units (color coded in orange, green, and blue respectively). Note, the progress of the project follows a curve, which results from the fact that the duration to remove spoil and bring concrete ring segments to the tunnel face increases with tunnel length. Such a dependence can be readily established as a function of the position along the length of the tunnel. There are many refinements that may be made to this model, to provide more accuracy and/or greater detail to allow decisions to be made about equipment types to be employed. Other attributes may be added, such as crew members, allowing these to be shared between different work units concurrently.

To illustrate the visual power of these models, consider the problem where two separate crews will be employed for tunneling, each starting at the same point but heading in opposite directions. If crewperformance records indicate that $1 \mathrm{crew}$ tends to operate about $50 \%$ faster than the other then we would want to find a starting location that would minimize the total project duration. Figure 9 shows the model for a $60 \mathrm{~m}$ tunnel with the two crews starting at the midpoint, with the slower crew heading to the left and the faster crew heading to the right. It can be seen from this chart that the faster crew should probably start $3 \mathrm{~m}$ or $6 \mathrm{~m}$ to the left of the midpoint to minimize the project duration - both choices could be tested quickly. Alternatively, an additional dimension could be added to the model representing starting the crews at different positions along the tunnel length, thus providing an automated sensitivity analysis of 
project duration versus starting point for the crews.

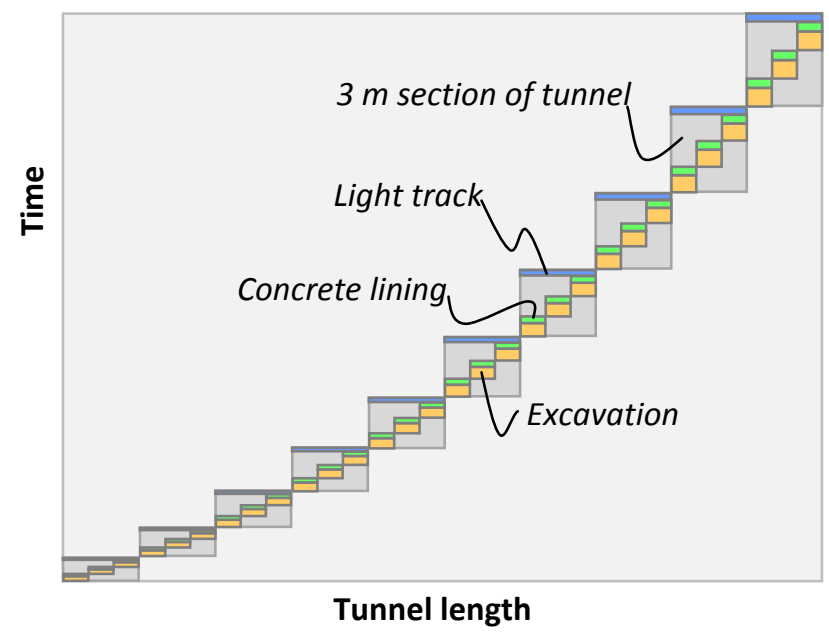

Figure 8: Sewer-tunnel model for the first 30 meters summarized to an intermediate level of breakdown

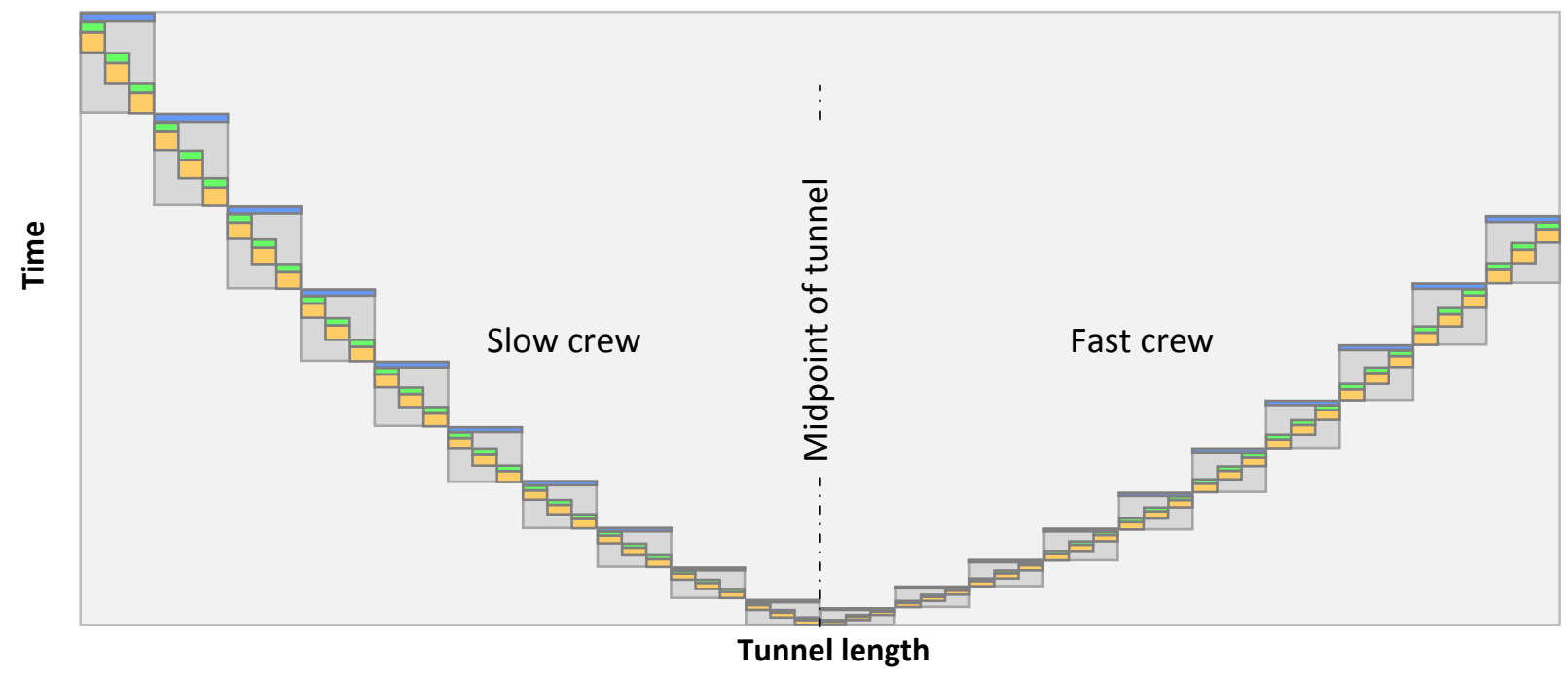

Figure 9: Tunnel model with two crews starting at centre and heading in opposite directions

\section{UNDERGROUND ELECTRIC CABLE LAYING PROJECT}

Figure 10 shows the completed Foresight model of an electric cable laying project typical of the type of process that would be modeled using linear scheduling except that it includes some logical features that are beyond conventional linear scheduling methods.

The project comprises four main tasks: excavate trench; shore trench; lay cable; and backfill, each of which is color coded. The smaller sized work units represent work on $1 \mathrm{~m}$ lengths of the project while the larger work units are essentially summaries of each task. Important feature of this model are:

- There are two crews available for excavation work and two crews available for shoring. The first excavation crew is faster than the second and will leap frog them when they reach their starting point.

- Shore trench has two constraints relative to Excavate trench, a minimum and a maximum permissible distance. The minimum working distance is for safety and to prevent interference between the crews. The maximum distance is to minimize the chances of the trench collapsing before being 
shored. In this example, the Shore trench crew must spend some time idle to ensure that the minimum distance constraint is not violated. If they operated considerably slower then the excavation crew would have had to spend time idle to ensure the maximum distance buffer was not violated.

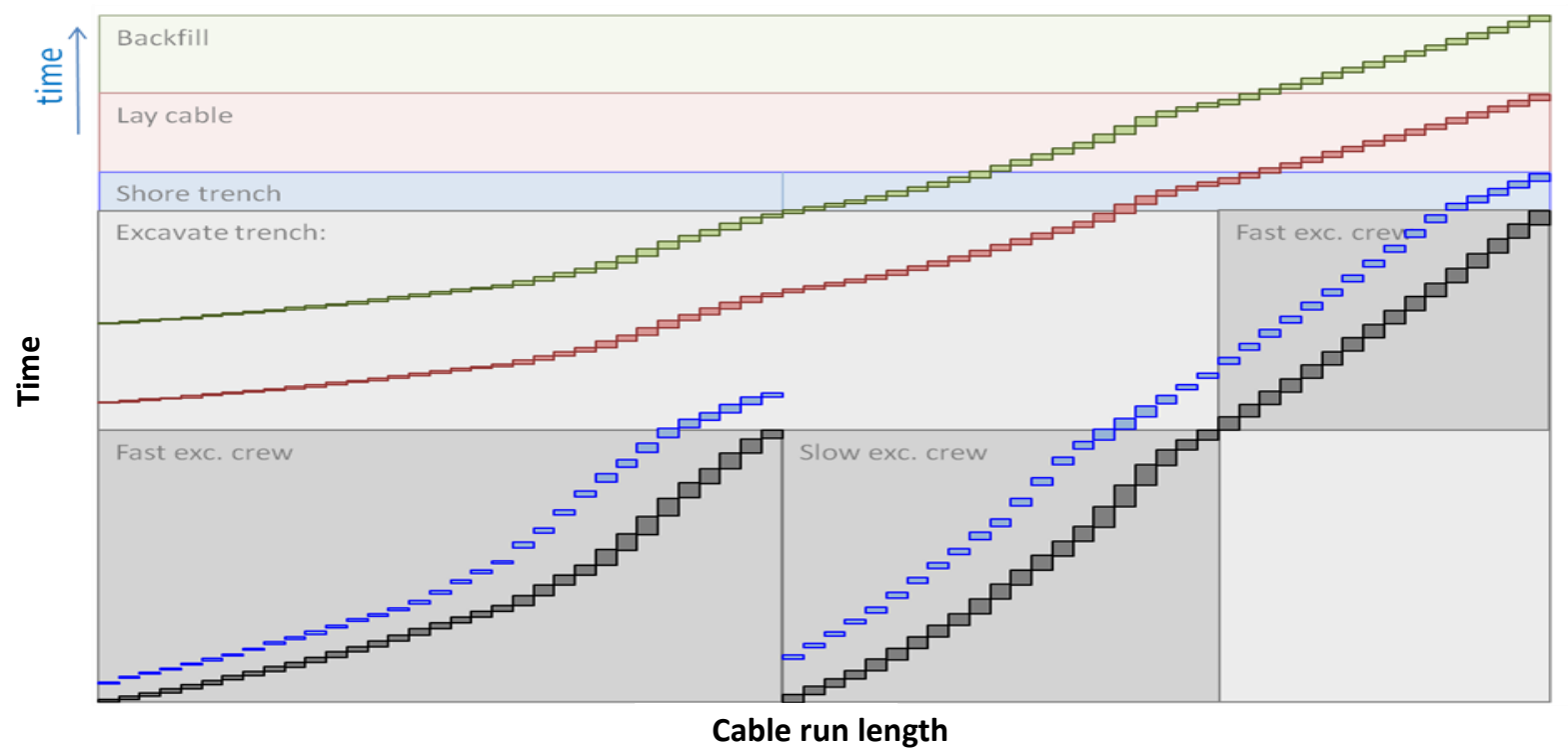

Figure 10: Underground electrical cable laying project

A constraint is imposed on both Lay cable and Backfill that prevents gaps between their work units within the model (effectively meaning they cannot spend time idle). This constraint reduces the amount of time their crews are employed on the project. As a consequence, Lay cable and Backfill are forced to start late.

\section{CRITICAL PATH TYPE ANALYSIS}

The final example illustrates the application of Foresight to the construction of a Bus Stop, which involves non-repetitive construction work, and is typical of the type of project that would be modeled using the Critical Path Method (CPM). Figure 11 shows the result of breaking down the project into a hierarchy of work units (in this case operating within the time and direct-costs dimensions) and with all constraints resolved. This model, as with any developed using Foresight, can be used conveniently to predict expected progress and monitor actual progress of work, functions crucial to effective project planning. Moreover, unlike conventional CPM, this can be done at any level within the structure of the model.

Figure 12, for example, shows projected progress for the Bus Stop project at the third level in the models hierarchy. Projected progress is indicated by the lines which measure (in this case as direct costs (\$)) the amount of a work unit expected to be completed at any given time. These curves can be used to gain insight into the expected progress of the project, and provide a template against which to measure actual progress on site. Progress curves are generated by the model by tracing the accumulation of work completed by work units over time. Consider the work unit Structural shown in Figure 9. This represents about $\$ 6,000$ in direct costs, made up from the direct costs of its constituent work units, and follows a classic $S$-curve in form.

Progress curves could have been plotted at any level in the model's hierarchy from the highest to the lowest levels. Progress curves at a higher level are good for gaining an understanding of the general rate of progress of the model. In the case of direct costs, this would be useful for determining, among other things, an appropriate line-of-credit required to finance the project. In contrast, progress curves plotted at the lowest level in the project are good for analyzing the likely continuation and impact of any delays to the actual progress of work on site, and for determining what effort is required to bring the project back on target in such events. 


\section{Flood}

$\uparrow$ Direct Costs $(\$)$

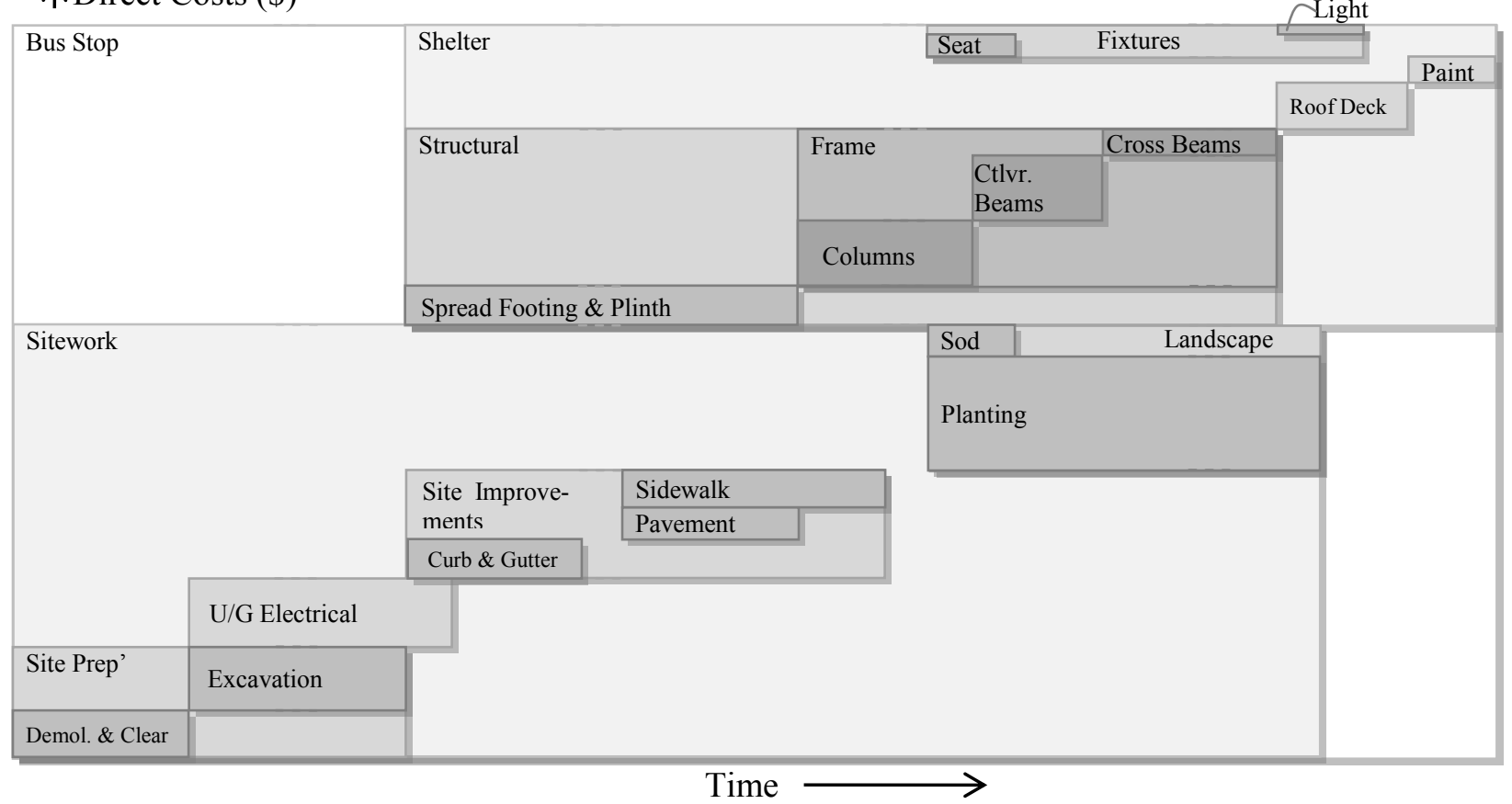

Figure 11: Foresight model of a bus stop construction project

\section{个Direct Costs $(\$)$}

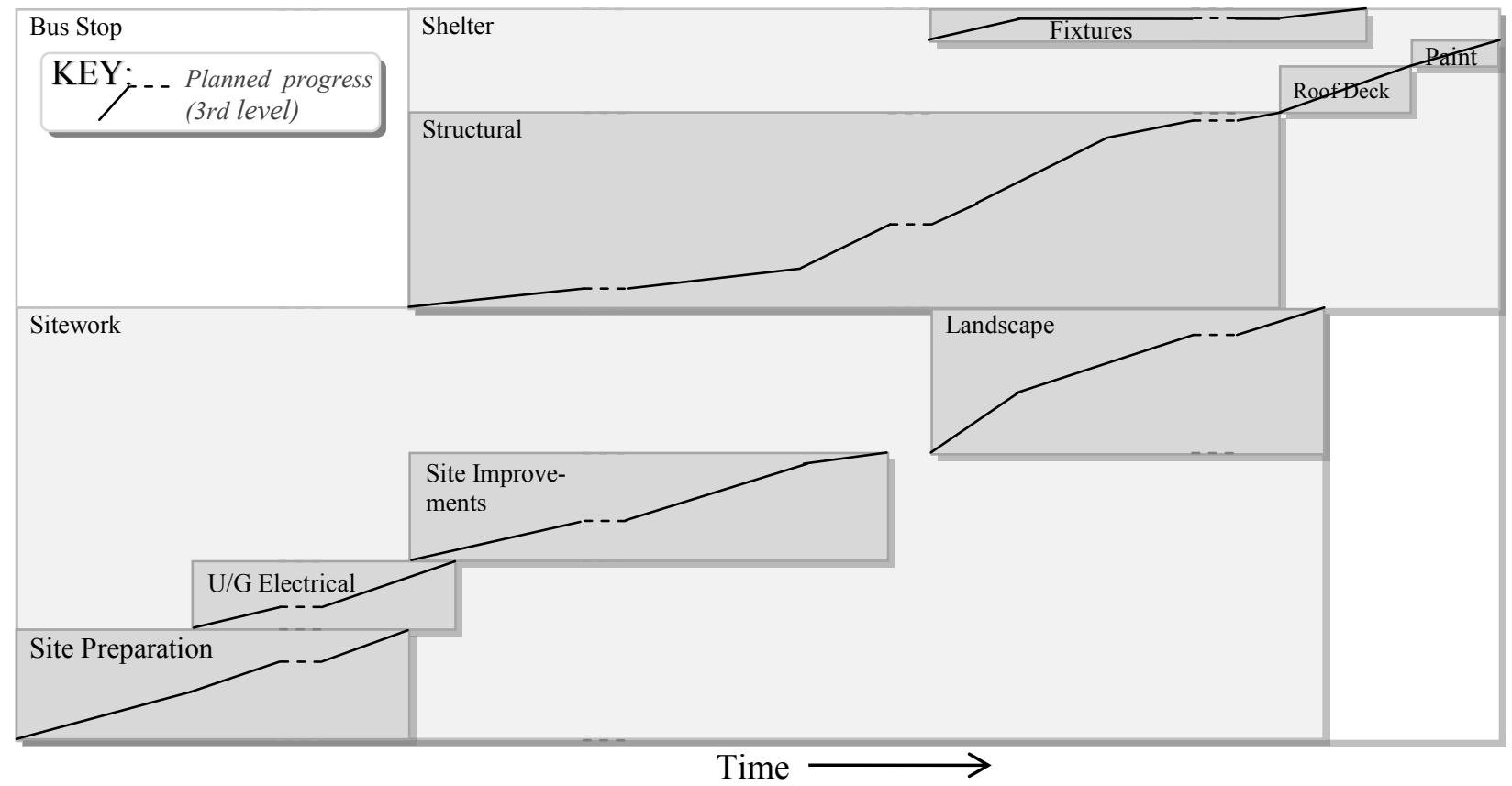

Figure 12: Bus stop construction model showing progress at the $3^{\text {rd }}$ level of breakdown

\section{CONCLUSIONS}

The paper has proposed a new technique, Foresight, that can be used to model construction processes that integrates the advantages of CPM, linear scheduling, and discrete-event simulation, along with hierarchical and interactive approaches to model development and analysis. The principles upon which Foresight is based provide it with the versatility necessary to model the broad spectrum of construction 
projects that until now have required the use of several different modeling tools. The resultant models are highly visual in form, representing the progress of work within the model structure. This provides insight into how the design of a process will impact its performance, and suggests ways of optimizing project performance.

Work is on-going developing detailed models using this method for a variety of project types. The objective of these studies is to determine the successes and limitations of the proposed planning method in the real-world, and to determine refinements that will increase its value as a modeling tool. The Foresight system as discussed is conceptual and work is underway implementing it for general use within the construction industry, using an object-oriented programming language since Foresight models are inherently object-oriented in structure.

\section{REFERENCES}

Ceric, V. 1994. "Hierarchical Abilities of Diagrammatic Representations of Discrete-Event Simulation Models." In Proceedings of the 1994 Winter Simulation Conference, eds. J. D. Tew, S. Manivannan, D. A, Sadowski, and A. F. Seila, 589-594. Piscataway, New Jersey: Institute of Electrical and Electronics Engineers, Inc.

Flood, I., R.R.A. Issa, and W. Liu. 2006. "A New Modeling Paradigm for Computer-Based Construction Project Planning." In Proceedings of the Joint International Conference on Computing and DecisionMaking in Civil and Building Engineering, ed. Hugues Rivard, 1-11. Montreal, Canada: ASCE.

Hajjar, D., and S.M.AbouRizk. 2002. "Unified Modeling Methodology for Construction Simulation." Journal of Construction Engineering and Management, ASCE, 128(2): 174-185.

Halpin, D.W., and R.W. Woodhead. 1976. Design of Construction and Process Operations. New York, NY: John Wiley and Sons, Inc.

Harris, R.B., and P.G. Ioannou. 1998. Scheduling Projects with Repeating Activities. In Journal of Construction Engineering and Management, ASCE, 124(4): 269-276.

Huber, P., K. Jensen, and R.M. Shapiro. 1990. "Hierarchies of Coloured Petri Nets." In Proceedings of the 10th Int. Conf. on Application and Theory of Petri Nets, ed. Grzegorz Rozenberg, 313-341. Bonn, Germany: Springer-Verlag.

Issa, R.A., I. Flood, and W. O'Brien, (Eds.). 2003. 4D CAD and Visualization in Construction: Developments and Applications. Steenwijk, Netherlands: A. A. Balkema.

Koo, B., and M.Fischer. 2000. "Feasibility Study of 4D CAD in Commercial Construction." Journal of Construction Engineering and Management, ASCE, 126(4): 251-260.

Matilla, K.G., and D.M., Araham. 1998. "Linear-Scheduling: past research efforts and future directions." Engineering, Construction, and Architectural Management, Blackwell Science Ltd, 5(3): 294-303.

Sawhney, A., S.M. AbouRizk, and D.W. Halpin. 1998. "Construction Project Simulation using CYCLONE." Canadian Journal of Civil Engineering, 25(1): 16-25.

\section{AUTHOR BIOGRAPHY}

IAN FLOOD is Holland Professor in the Rinker School, College of Design, Construction and Planning at the University of Florida. He received his Ph.D. from the University of Manchester, UK, on parallel computing techniques applied to the simulation of construction processes. He serves as a Specialty Editor for both the Journal of Computing in Civil Engineering, ASCE, and the Journal of Advanced Engineering Informatics, Elsevier. He has published over 120 refereed articles on the subjects of simulation modeling and intelligent computing applied to civil engineering. His email is $<$ flood@ufl.edu $>$. 